\title{
Echo Cancellation Research of Channel Estimation based on PN Sequence
}

\author{
Yongqin Zhou \\ (College of Electrical \& Electronic Engineering. Harbin University of Science \& Technology \\ Harbin150080,China) \\ Email: zyqemail@163.com \\ Ming Ge and Shuzhi Ji \\ (College of Electrical \& Electronic Engineering. Harbin University of Science \& Technology \\ Harbin150080,China) \\ Email: \{ geming2020, alvinddj\}@163.com
}

\begin{abstract}
For the problem of estimation sequence effect on channel estimation accuracy and echo cancellation effect, this paper, based on the basic principle of echo cancellation, analyses the effect of $P N$ sequence mechanism and the correlation on the channel estimation parameters. Comparing with using the input signal itself as the estimation sequence. With the input signal OFDM, the results of simulation and actual operation show that the method can increase both the accuracy of channel estimation and echo cancellation effect effectively.
\end{abstract}

Index Terms-PN sequence, channel estimation, echo cancellation, correlation

\section{INTRODUCTION}

In long distance communications and digital cochannel repeater, the receiver will receive a strong multipath echoes which seriously affect the signal quality of communications, because the physical location relationship of the transmitting antenna and the receiving antenna add the refraction and reflection of the ground. The echo is interference signal with frequency through multiple he channel attenuation and returns to the input. Conventional duplexers or filters can filter out the interference signal outside the work band, it is difficult to filter out the signal in the work band. he echo cancellation is designed to eliminate the echo effect on the system.

The basic idea to achieve echo cancellation system is that leading the error signal and the output signal to the filter through the pseudo channel .Through estimating the actual attenuation channel and adjusting the filter parameters, the signal similar to echo is generated in the filter, which is to offset the echo. Obviously the degree of echo cancellation is affected by the accuracy of the channel estimation. The estimated sequence is needed in the estimated channel. Moreover, the correlation with other signals and auto-correlation of the sequence are required as small as possible ${ }^{[1]}$. General system is often estimated, using the input signal as the estimated sequence to estimate channel ${ }^{[2]}$,the relevance of the input signal is not ideal so that the accuracy of estimated channel is not satisfied, therefore, many researchers have proposed using the PN sequence of good correlation as the estimated sequence to estimate the channel ${ }^{[3 \sim 5]}$.The existing literature show that using PN sequence as the estimated sequence is feasibility and the results are produced by combining with all kinds of adaptive algorithm, the problem of the estimated sequence effect on the estimated sequence accuracy is not analyzed .The paper combine the PN sequence with the adaptive algorithm, analyze the mechanism of the PN sequence and the reasons of estimated channel accuracy, and apply in the echo cancellation system. The results of simulation and practical operation after using the OFDM signal as the input signal prove that using the PN sequence as the input signal can effectively increase the accuracy of the estimated sequence, improve both the effect of echo cancellation and the communication quality.

\section{PRINCIPLE OF ECHO CANCELLATION}

In the digital repeater channel estimation and the principle of echo cancellation are shown in Figure 1. Among them, the sequence of echo cancellation is $\mathrm{h}^{\mathrm{H}}(\mathrm{n})$, the input signal is $s(n)$, and the value of echo estimated signal out of filter is $y(n)$. The figure shows that the input signal $s(n)$ superposition with the echo interference after subtraction the estimated value is very close to the $y(n)$ as $y(n)$ is the estimated value of the echo. Estimated sequence generated by the estimated sequence generator, one channel of which all the way enter the weight coefficient update module and the other channel superposition with the e(n)are viewed as the output signal to launch out. At the same time $u(n)$ is introduced to the adaptive algorithm module to estimate echo. The $\mathrm{e}(\mathrm{n})$ is generated by the estimated value of $y(n)$ subtraction with the $d(n)$ of next time continuously offset the echo inference. First order weights of filter can simulate a fading channel to offset the echo, the more order weights is designed, the effect of echo cancellation is the better. As the hardware resources are limited and the

Natural science foundation of Heilongjiang province,

The key technique research on low power line of OFDM system

Project Numbet:F200914 
interference is weak after 48 decay channel, the order is designed to 48 .

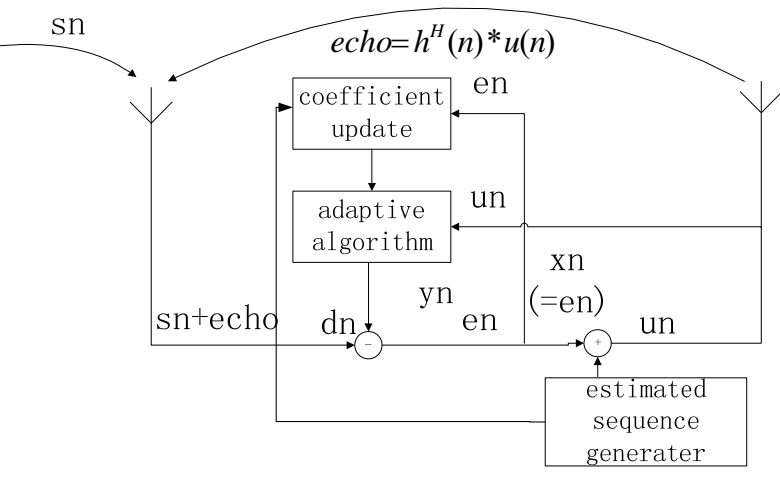

Figure 1 echo cancellation schematic of the adaptive filter

The algorithm of least mean square $\mathrm{LMS}^{[5,6]}$ can be used in the adaptive filter. The formula of LMS algorithm is:

$$
\begin{aligned}
& y(n)=w^{H}(n) x(n) \\
& e(n)=d(n)-y(n) \\
& w(n+1)=w(n)+2 \mu e(n) x(n)
\end{aligned}
$$

$\mathrm{y}(\mathrm{n})$ is the filter output, $\mathrm{e}(\mathrm{n})$ is the error signal, $\mathrm{w}^{\mathrm{H}}(\mathrm{n})$ presents the of each order weights, $\mathrm{x}(\mathrm{n})$ is the filter input, $\mu$ is the step length, increasing the value of which can increase the amount of the signal accumulation each time, reduce the system accuracy and the system convergence, and lower the echo cancellation effect. Therefore, to select a suitable $\mu$ value, requires repeated testing on the machine. The article is drawn by debugging that the effect is ideal when the $\mu$ value is around 0.1 . Echo interference signal is expressed as:

$$
e c h o(n)=h^{H}(n) x(n)
$$

\section{THE MECHANISM OF PN SEQUENCE}

PN sequence is a pseudo-random code, which has similar properties of random signals. Taking any two different times to compare are not completely similar, so using it to estimate the channel is less prone to confuse the two signals. In the other words, that is less to prone to interfere with each other and produce false positives. Using $\mathrm{M}$ sequence as the estimated sequence in the article, which is one kind of PN sequence. the input signal $x(n)$ itself isviewed as the estimated sequence in traditional systems. After the estimated sequence is replaced by the PN sequence, the formula is expressed as:

$$
\begin{aligned}
& y(n)=w^{H}(n) u(n) \\
& e(n)=d(n)-y(n)
\end{aligned}
$$

$$
w(n+1)=w(n)+2 \mu e(n) p(n)
$$

It can be seen that, $x(n)$ in the original (3) is replaced by $p(n)$ and $u(n)$ instead of $x(n)$ in the formula 1 . The signal $u(n)$ is the filter input, which is the sum of the signal $x(n)$ and $p(n)$ used in the estimated channel. Then echo interference signal is expressed as:

$$
\text { echo }(n)=h^{H}(n) u(n)
$$

The (5) (8) are deduced to explain the mechanism of PN sequence. Though weight coefficients of the filter should be 48 bands, here only the first two bands are listed in the derivation of weights, $2 \mu$ is a constant that can be ignored in the derivation.

$$
\begin{aligned}
u(n)= & x(n)+P(n) \\
= & \{x(n)+P(n)] \\
& ,[x(n-1)+P(n-1)]\}^{H} \\
y(n) & =w^{H}(n) u(n) \\
= & w(n-1)[x(n)+P h(n)] \\
& +w_{2}(n-1)[x(n-1)+P h(n-1)] \\
d(n)= & s(n)+e c h o(n) \\
= & s(n)+h^{H}(n) u(n) \\
= & s(n)+h_{1} \times[x(n)+P n(n)] \\
& +h_{2} \times[x(n-1)+\operatorname{Pn}(n-1)]
\end{aligned}
$$

$$
\begin{aligned}
e(n) & =d(n)-y(n) \\
= & s(n)+\left(h_{1}-w_{1}(n-1)\right)[x(n)+P n(n)] \\
& +\left(h_{2}-w_{2}(n-1)\right)[x(n-1)+P n(n-1)]
\end{aligned}
$$

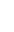




$$
\begin{aligned}
w_{1}(n) & =w_{1}(n-1)+e(n) P n(n) \\
= & w_{1}(n-1)+s(n) P(n) \\
& +\left(h_{1}-w_{1}(n-1)\right) x(n) P n(n) \\
& +\left(h_{1}-w_{1}(n-1)\right) \operatorname{Pn}(n) P(n) \\
& +\left(h_{2}-w_{2}(n-1)\right) x(n-1) P(n) \\
& +\left(h_{2}-w_{2}(n-1)\right) \operatorname{Pn}(n-1) P n(n)
\end{aligned}
$$

$$
\begin{aligned}
w_{2}(n)= & w_{2}(n-1)+e(n) P n(n-1) \\
= & w_{2}(n-1)+s(n) P n(n-1) \\
& +\left(h_{1}-w_{1}(n-1)\right) x(n) P n(n-1) \\
& +\left(h_{1}-w_{1}(n-1)\right) \operatorname{Pn}(n) P n(n-1) \\
& +\left(h_{2}-w_{2}(n-1)\right) x(n-1) P n(n-1) \\
& +\left(h_{2}-w_{2}(n-1)\right) \operatorname{Pn}(n-1) P n(n-1)
\end{aligned}
$$

In the first $2,3,5,6$ term of the formula 13 , the accumulation of each will fluctuate and not always increase as $\operatorname{Pn}(n) * \operatorname{Pn}(n)$ and the multiplier are not related. But in the item 4, the auto correlation of $\operatorname{Pn}(n)$ and $\operatorname{Pn}(n)$ is very good, and they must be positive after multiplying, the sum of $\operatorname{Pn}(n) * \operatorname{Pn}(n)$ will always be cumulative. When the condition of $h_{1} \geq w_{1}(n-1)$ is satisfied, it makes the value $w_{1}(n)$ has been increased, $w_{1}(n)$ and $w_{1}(n-1)$ is the weight coefficient of the same order at different times. Therefore, $\mathrm{w}_{1}(\mathrm{n}-1)$ also increase. The subtraction value of $\mathrm{h} 1$ and $\mathrm{w}_{1}(\mathrm{n}-1)$ makes $\mathrm{w}_{1}(\mathrm{n})$ close to $\mathrm{h}_{1}$, but when the condition of $\mathrm{h}_{1} \leq \mathrm{w}_{1}(\mathrm{n}-1)$ is satisfied, it makes $\mathrm{w}_{1}(\mathrm{n}-1)$ decreased, The subtraction value of $\mathrm{h} 1$ and $\mathrm{w}_{1}(\mathrm{n}-1)$ makes $\mathrm{w}_{1}(\mathrm{n})$ close to $\mathrm{h}_{1}$. In the same way, it makes $\mathrm{w}_{2}(\mathrm{n})$ close to $\mathrm{h}_{2}$ in the formula 14. Thus that produces the estimated signal of $y(n)$ that is very close to echo. They offset each other to achieve the effect of echo cancellation.

If using the signal $x(n)$ as the estimated sequence, $\mathrm{x}(\mathrm{n})$ does not have randomness. The input signal multiply other signals or their own signal at different times, the correlation of which is not small. We do analog analysis for the formula 13 find that the weight coefficient fluctuates, reduce the accuracy of the fading channel estimation and affect the results of echo cancellation. Therefore the simulation can take an absolute value less than 1 of a number two as the attenuation coefficient.

IV SIMULATION ANALYSIS
OFDM(Orthogonal Frequency Division Multiplexing)that is orthogonal frequency division multiplexing is used as the received signal, which is one of MCM Multi-Carrier Modulation. The technology is widely used in a variety of digital transmission and communication because it is easy implement with other multiple assess methods, efficiency of high spectrum. MATLAB is used in the simulation, and the input OFDM signal is a "normalized" approach. The each signal of maximum absolute value is divided by each signal value, the result of which is within plus or minus 1 . Because of the above principle and (5) (8), we edit $M$ files, create simulation models, estimate the channel with the PN sequence, the simulation results is shown in Fig. 2 6.

Fig. 2 shows the OFDM modulated signal power spectrum, it can be seen the signal OFDM modulated have good flatness and bandwidth, small burr-band and smooth signal. OFDM signal modulated after transmission may be disturbed, due to the impact of echo signal. Since echo attenuation paths are infinite, echo signal is difficult to fully simulate. Thus 0.5 and 0.51 are selected, then the output signal after delay is multiplied by the sum of the two attenuation coefficients to simulate the echo. Echo interference power spectrum are shown in Fig.3, and the modulated signal OFDM which is unprocessed is affected by the echo interference, which is shown in Fig. 4. It can be seen from Fig. 4 that the modulated signal is serious distortion, thus it will affect the communication quality. For example, answering the call will hear the echo of your voice, and listening to the radio will hear intermittent noise, sometimes the signal which can not be restored cause the communication interruption. Therefore, the echo interference signal must be processed.
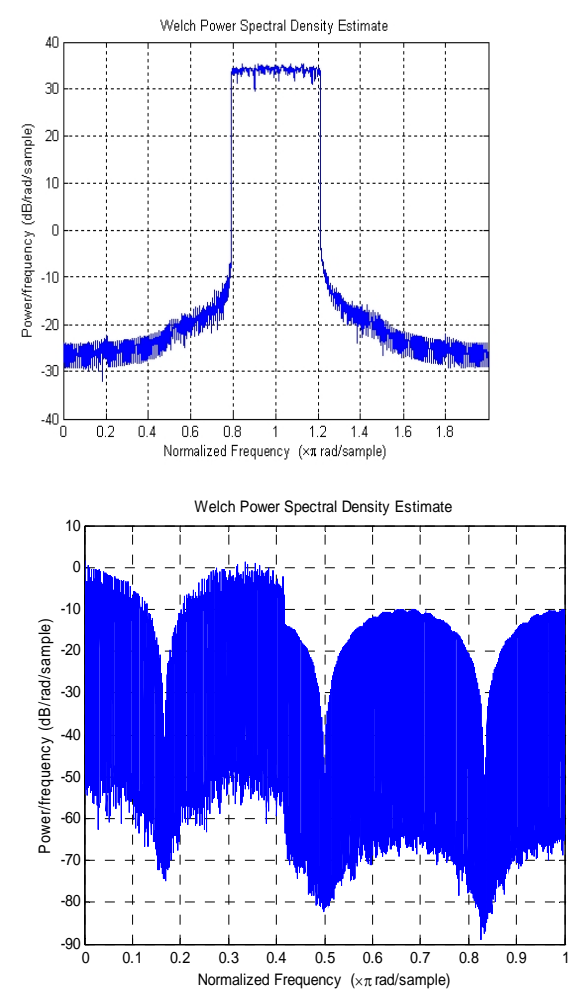

Figure 3 power spectrum of echo interference 
The way of echo cancellation is required to eliminate echo interference, using PN sequence as the estimated sequence produce estimated echo is shown in Fig. 5. It can be seen that it is very close to Fig. 3. The tendency effect of offsetting is better than the receive signal e(n), which can be seen in Fig. 6. Figure a shows the processed signal e(n)gradually approaches $s(n)$, the tendency has good results, which can be seen through its back-end amplification (Figure b).

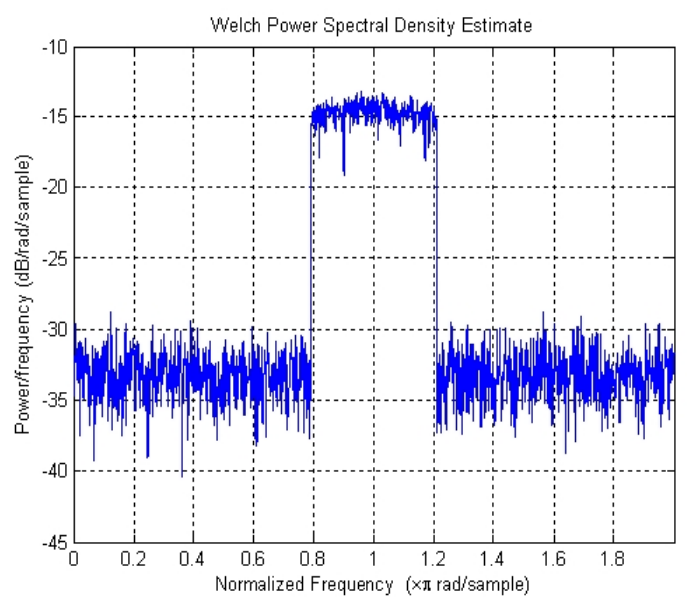

Figure 4 power spectrum of OFDM signal interfered

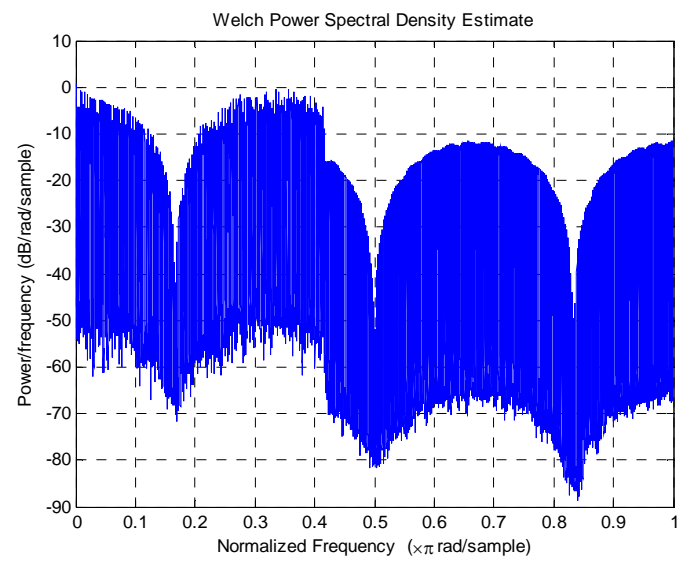

Figure 5 estimated echo power spectrum of $\mathrm{PN}$ sequence

estimation for channel

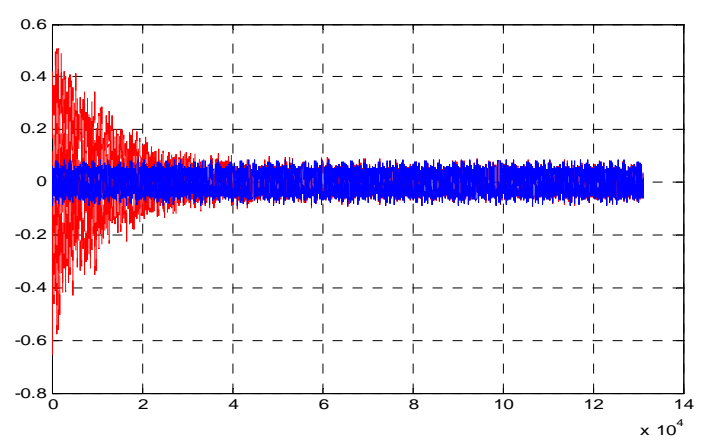

(a)the overall figure

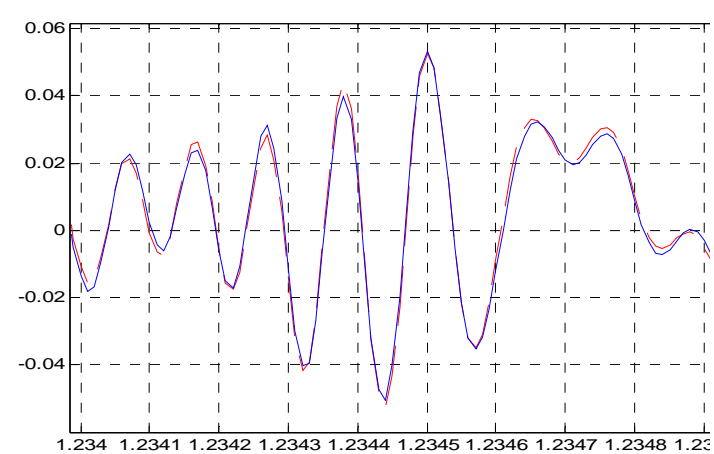

(b) back-end enlargement

Figure 6 signal simulation timing diagram of PN sequence estimation for channel

The output power spectrum after echo cancellation is shown in Fig.7, contrast to the Fig 4, it can be seen that the signal quality has been greatly improved.

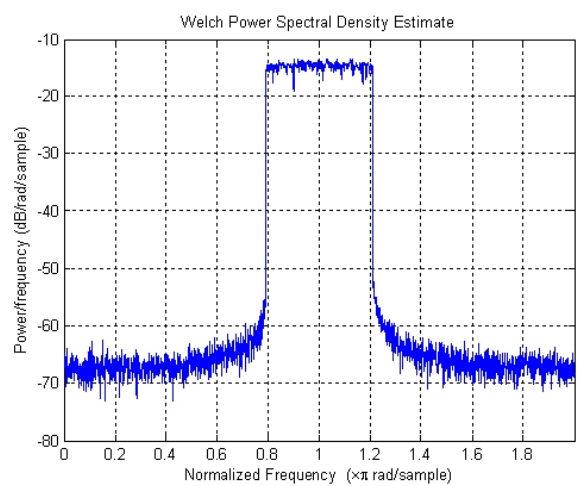

Figure 7 the channel's output signal of PN sequence estimation

When the PN sequence introduced to the system is viewed as the estimation sequence, though the accuracy is improved, and PN sequence is also similar to the white noise of a random signal which is shown in the Fig. 8

, the introduction of PN sequence is equivalent to add noise to the system. The signal quality may be affected because of a little bigger of the PN sequence. So the PN sequence is needed to adjust to a appropriate value, the simulation waveform is observed by repeatedly adjusting the value of $\mathrm{PN}$

The value of PN sequence should be as small as possible in condition that the system accuracy is not affected until the output waveform achieves the desired results, which is shown in the Fig. 7

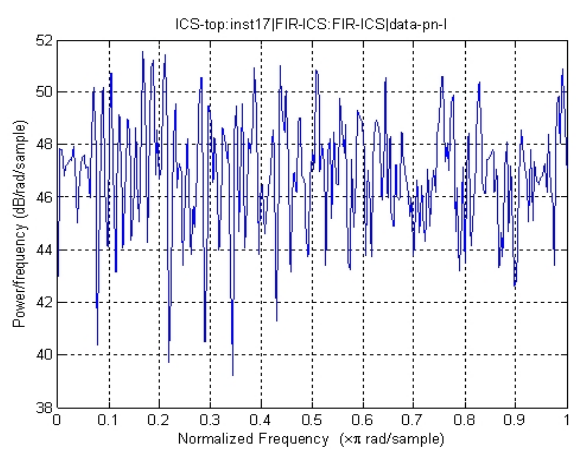

Figure 8 the power spectrum of the PN sequence 
In order to observe the effect of using the PN sequence as the estimation channel, it can make a contrast to the result of using the input signal as the estimation sequence. According to the (1) to (4),M files of using the PN sequence as the estimation sequence is written in the same way, the simulation result is shown in Fig. 7 to 8 .

Fig. 7 is compared with Fig. 5 for estimated echo, the latter is closer to echo interference(Fig. 3). Matrix norm can be introduced to the comparison. when PN sequence is used to estimate channel, the error norm of estimated echo matrix(expressed as Y1) and echo interference Matrix(expressed as E)is \| E-Y1 \|= $8.247098511325040 \mathrm{e}+03$ if a number of 70,000 is taken out, to an contrast, when the input is used as the estimated consequence the error norm of echo matrix (expressed as Y2) and echo interference mattix(expressed as E) is $\|\mathrm{E}-\mathrm{Y} 2\|=9.749038213357030 \mathrm{e}+03$, obviously, the fomer norm is smaller. The smaller the value is, the closer the value of the previous subtration two matrixes and the higher the estimation accuracy is, which shows that the estimation of using the PN sequence as the estimated sequence is close to the echo interference. When the input is used as the estimated sequence the tendency effect of echo cancellation signal e(n)and the received signal after echo cancellation is shown in Fig. 8, contrast to Fig. 6, using PN sequence to estimate channel is better.

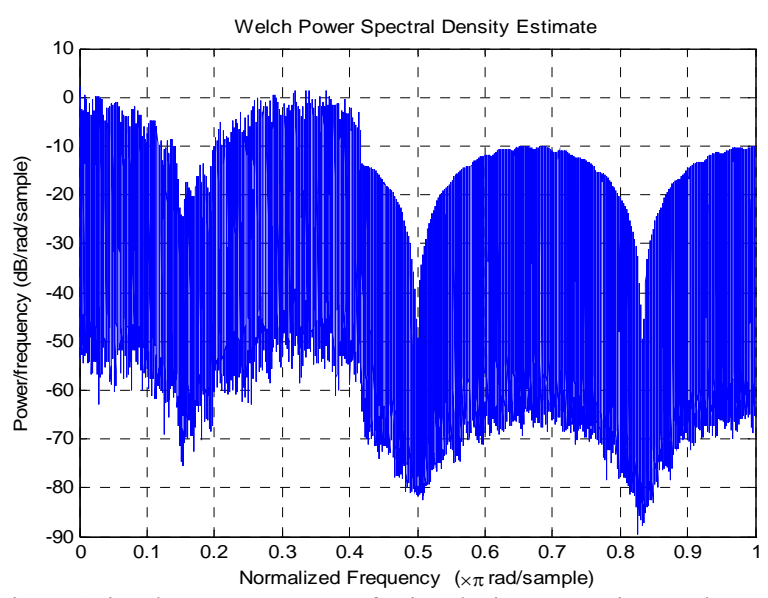

Figure 9 signal power spectrum of using the input to estimate channel

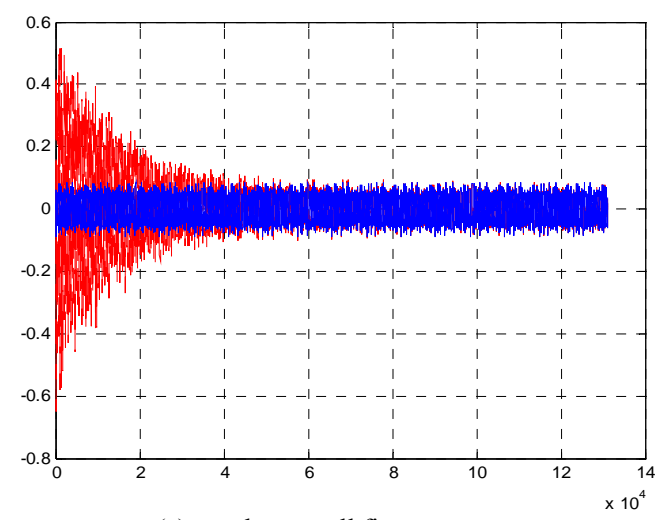

(a) the overall figure

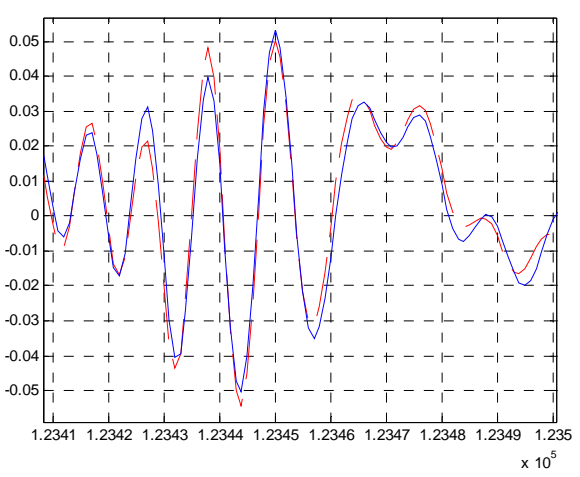

(b)back-end Enlargement

Figure 10 signal simulation diagram of using the input to estimate channel

\section{V.ECHO CANCELLATION IMPLEMENTATION AND DEBUGGING}

FPGA can be used in the echo cancellation circuit and hardware development platform is designed by the Altera, Verilog language is used to write programs in the QuartusII ,which is downloaded to the FPGA. Then it generates an adaptive filter in the FPGA and a similar signal to offset echo cancellation. PN sequence can use the MIF file generated by MATLAB to store in the ROM produced in the FPGA and read to the signal source.

After the program is integrated, implemented, compiled, simulated, it can be downloaded to the FPGA to debug. The effect of PN sequence for the output must be paid attention when debugging. In the project, $\mathrm{PN}$ sequence's MIF files generated by MATLAB is discarded a cut-off bit to reduce value of PN sequences, so that the impact of the PN sequence is reduced. In the same way, PN sequence's cut-off bit should gradually be adjusted to avoid being smaller of the PN sequence, thus it makes the system self-excited . spectrum analyzer of HEWLETT PACKARD 8594E is used to debug and it does not have the function of picture collection. The following two pictures are photographed by camera. The output signal of debugging result is using the input signal itself and PN sequence as the estimated sequence which is separately shown in the Fig. 11 and Fig. 12. The OFDM signal power spectrum is captured by the spectrum when the step $\mu$ is 0.1 .

Fig. 11 and Fig.12 show that echo interference obtained by using the PN sequence to estimate channel is smaller, three key indicators of offsetting effect can be verified by the spectrum analyzer and signal source in the figure, the three indicators are isolation, band fluctuations and stray out of band. Isolation is power ratio of the input and the output, it reflects the input amplification range in the system. the greater the isolation is ,the bigger the system allows the maximum output power. Band fluctuations and stray out of band reflect the interfered level of signal, the smaller they are, the less the interference is. Using the signal itself and the PN sequence as the estimated sequence make a technical comparison, which is shown in the table 1 . 


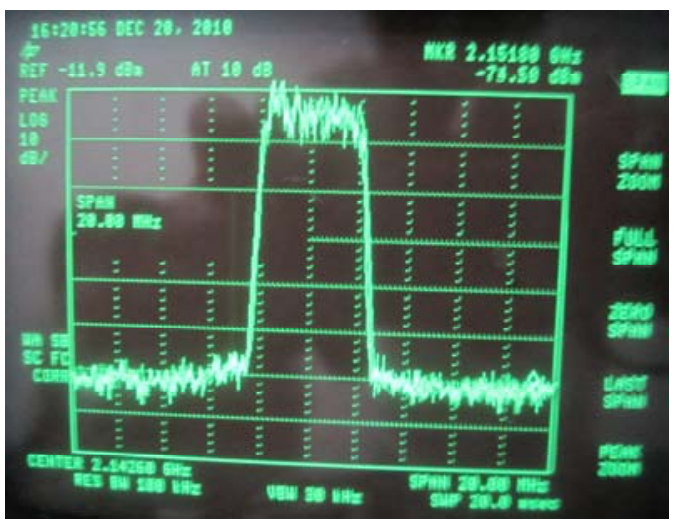

Figure 11 the output signal of using the input signal to estimate channel

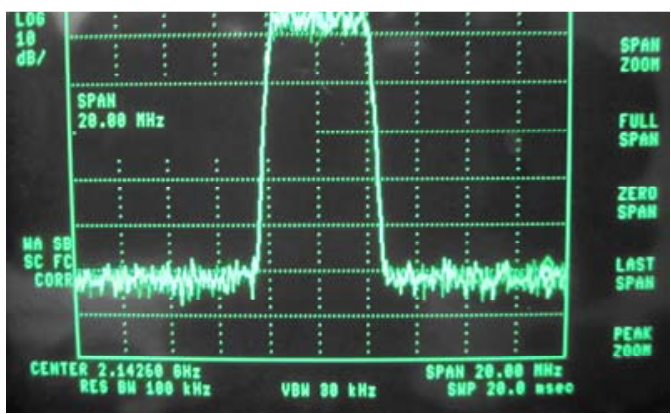

Figure 12 the output signal of using the PN sequence to estimate channel

Table 1 technical indexes comparison table

\begin{tabular}{|c|c|c|c|}
\hline $\begin{array}{c}\text { kind of } \\
\text { estimation } \\
\text { sequence }\end{array}$ & $\begin{array}{c}\text { increased the } \\
\text { maximum } \\
\text { isolation }\end{array}$ & $\begin{array}{c}\text { minimum } \\
\text { fluctuation } \\
\text { of band }\end{array}$ & $\begin{array}{c}\text { minimum } \\
\text { stray of } \\
\text { band }\end{array}$ \\
\hline input signal & $31 \mathrm{~dB}$ & $1 \mathrm{~dB}$ & $-62 \mathrm{~dB}$ \\
\hline $\begin{array}{c}\mathrm{PN} \\
\text { sequence }\end{array}$ & $37 \mathrm{~dB}$ & $0.6 \mathrm{~dB}$ & $-68 \mathrm{~dB}$ \\
\hline
\end{tabular}

It can be seen from Table 1, using the PN sequence as the estimated sequence cause the system isolation to increase .this is due to the enhanced effect of echo cancellation and the decreased disturbance, which make the output effect on the input decreased, the output power increased, and isolation enhanced as using the PN sequence to estimate the channel improve the estimation accuracy and enhance the filter's effect the band fluctuation and stray reduce, using PN sequence to estimate the channel is mentioned in the simulation above, the glitch is greatly reduced, indicating that the system performance achieve the desired effect.

\section{CONCLUSIONS}

Echo cancellation effect is subject to the accuracy of the channel estimation, but the channel estimation accuracy is affected by serial correlation. PN sequence based on echo cancellation way of using a PN sequence to estimate channel have good auto-correlation and cross- correlation can improve both the channel estimation accuracy and the echo cancellation effect. This echo cancellation method can be used in long-distance communication or the digital Same frequency repeater and high quality communication signals can be obtained.

\section{REFERENCES}

[1] Yongqin Zhou,Shuzhi Ji,Xudong Wang ,Ming Ge,Echo "Cancellation Technology based on PN Sequences",25-26 Dec.2010

[2] Jin-kyu Hong, Young-woo Suh, Jin-yong Choi and Jong-Soo Seo, "Echo Canceller for On-Channel Repeaters in T-DMB System[J]”, Feb.17-20, 2008 ICACT 2008, pp1735-1738;

[3] Bowei Song,Lin Gui,Yunfeng Guan and Wenjun Zhang,"On Channel Estimation and Equalization in TDS-OFDM based Terrestrial HDTV Broadcasting System[J]", IEEE Transactions on Consumer Electronics, Vol. 51, No. 3,AUGUST 2005, pp790797;

[4] Xiaodong Wang."OFDM and its application to 4G.International Conference on Wireless and Optical Communication, 2005,4:69-74".

[5] Haodong Bai, "estimation method of wireless channel for OFDM based on PN sequence" North China Electric Power University, a maser's degree thesis, 2008

[6] Lihui Wang, Feng Sun, Qiang Ji,Yanling Hao. "LMS adaptive filter's algorithm applied in the data processing of FOG system" Systems and Engineering and Electronics,2010;

[7] Yuanjie Li, Lvxi Yang, Zhenya He. "Channel estimation and tracking based on implicit training sequence" Communications,2004;

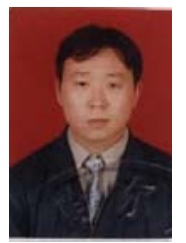

Yongqin Zhou was born in 1971, Henan province of China. He received M.S. degree in power electronics and electrical drivers from Harbin University of Science and Technology in 2003.

$\mathrm{He}$ is currently a vice-professor of the Harbin University of Science and Technology and a member of Automobile Electronic Engineering Center. Some of the published articles are Research on Power Train Source Matching for Singleaxle PHEV, 2008 IEEE Vehicle Power and Propulsion Conference, 2008, 9, and The Research and Realization for Passenger Car CAN Bus. Now he is doing the key technique research on low power line of OFDM system and supported by the natural science foundation of Heilongjiang province,

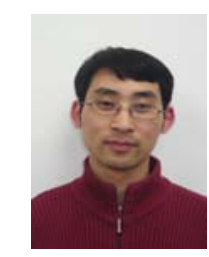

Ming Ge was born in 1983, Heilongjiang province of China.,He received B.S. degree in electrical engineering from Harbin University of Science and Technology, Heilongjiang, China, in 2008. He is a graduate student for a M.S. degree in Harbin University of Science and Technology, under the guidance of Dr. 
Shuzhi Ji was born in 1985, Heilongjiang province of China. He received B.S. degree in electrical engineering from Harbin University of Science and Technology, Heilongjiang, China, and M.S. degree in power electronics and electrical drives from Harbin University of Science and Technology, in 2011. His M.S. dissertation under the supervision of Dr. Zhou, was entitled "Research on System of Echo Filter Based on FPGA " 